\title{
Screening of Rice (Oryza sativa L.) Genotypes at Reproductive Stage for their Tolerance to Salinity
}

\author{
M. M. Islam*, M. H. Faruqe, M. S. Rana, M. Akter and M. A. Karim \\ Department of Agronomy, Bangabandhu Sheikh Mujibur Rahman Agricultural University, Gazipur- \\ 1706, Bangladesh.
}

*Corresponding author and Email: moshiul@bsmrau.edu.bd

Received: 22 April 2018

Accepted: 23 June 2018

\begin{abstract}
Soil salinity is one of the most devastating environmental stresses for rice production in the coastal areas of Bangladesh. Improvement in salt tolerance of rice is an important way for the economic utilization of coastal zones. An experiment was conducted at the vinyl house of the Department of Agronomy, Bangabandhu Sheikh Mujibur Rahman Agricultural University (BSMRAU), Bangladesh during December 2016 to April 2017 to determine the effects of different salinity levels on the yield and yield components of some rice (Oryza sativa L.) genotype sand finally, screening of rice genotypes for salt tolerance. The experiment containing four treatments was laid out in a complete randomized design with five replications. The treatments were four levels of saline water with electrical conductivities at control $\left(0.3 \mathrm{dSm}^{-1}\right), 5,10$ and $15 \mathrm{dSm}^{-1}$. Based on screening at germination stage, relatively salt-tolerant eleven genotypes with two check varieties were used in this experiment. The study showed that increase in salinity level significantly decreased yield and yield contributing characters of rice. However, among the eleven, three genotypes (Chapali, Patnai 23 and Soloi) were considered as moderately salt tolerant at $15 \mathrm{dSm}^{-1}$ on the basis of their yields and yield contributing characteristics such as plant height reduction, total tiller reduction, effective tiller reduction, reduction of fertile grains per panicle, grain yield and relative grain yield. Therefore, Chapali, Patnai 23 and Soloi might be recommended as moderately salt tolerant rice genotypes.
\end{abstract}

Keywords: Salinity level, Rice genotypes, Yield components, Tolerance.

\section{Introduction}

Rice (Oryza sativa L.) is an important cereal which is consumed as a staple food by more than half of the world's population (Ma et al., 2007). The world population is increasing rapidly and may reach 6.0 to 9.3 billion by the year 2050 (UNFPA, 2014), whereas the crop production is decreasing rapidly by the negative impact of various environmental stresses (Reddy et al., 2017). Therefore, it is now very important to develop stress-tolerant varieties to cope with this upcoming problem of food security. To assure food security in the rice consuming countries of the world, rice production should be increased by $50 \%$ in these countries by 2025 . Therefore, for the sake of food security the saline-prone area must be brought under intensive crop cultivation. Salinity is one of the major abiotic stresses that substantially limiting crop production and reduces the average yield of major crops by more than 50\% (Abdallah et al., 2016). The deleterious effects of salt stress on agricultural yield are significant, mainly because crops 
exhibit slower growth rates, reduced tillering and, over months, reproductive development is affected (Munns et al., 2008). Salinity primarily imposes on plants an osmotic stress and secondarily ion toxicity stress. Excess salt in the soil may adversely affect plant growth either through osmotic inhibition of water uptake by roots or specific ion effects. Specific ion effects may cause direct toxicity or alternatively, the insolubility or competitive absorption of ions may affect plant nutritional balances (Silva et al., 2008). Excess $\mathrm{Na}^{+}$in plant cells directly damages membrane systems and organelles, resulting in plant growth reduction and abnormal development prior to plant death (Quintero et al., 2007; Siringam et al., 2011). If excessive amount of salt enters into plant, the concentration of salt will eventually rise to a toxic level in older transpiring leaves causing premature senescence and reduced photosynthetic leaf area of a plant to a level that cannot sustain growth $(\operatorname{Rad}$ et al., 2012a).

Photoinhibition coupled with salinity stress causes serious damage to many cellular and physiological processes including photosynthesis, nutrient uptake, water absorption, root growth, spikelet formation, fertilization of florets, and cellular metabolism, which all obviously lead to yield reduction (Darwish et al., 2009). High salt concentration may lead to plant death and no yield.

More than 800 million ha of land throughout the world are salt-affected (FAO, 2008). Most of the southern districts of Bangladesh are under saline zones, which cover an area of 25-30 percent of the total arable land (Haque, 2006). The average crop yield is very low in the region, which is obviously due to salinity problems. Rice is generally sensitive to salinity (Moradi and Ismail, 2007) though rice varieties differ greatly in salinity tolerance (Kamyab-Talesh et al., 2014; Abdullah et al., 2016). Although rice is the predominant crop of Bangladesh, modern rice varieties tolerant to salinity are few in number. So, it is necessary to develop rice varieties against salt stress to meet the future food demand. The objectives of the present study was to provide information on the effect of salinity on yield and yield components of rice genotypes and finally screening of rice genotypes for salinity tolerance.

\section{Materials and Methods}

The field experiment was conducted at the Department of Agronomy, Bangabandhu Sheikh Mujibur Rahman Agricultural University (BSMRAU), Gazipur, Bangladesh during December 2016 to April 2017. The site is located at $24^{\circ} 0^{\prime} \mathrm{N}$ latitude and $90^{\circ} 25^{\prime} \mathrm{E}$ longitude at an elevation of $8.4 \mathrm{~m}$ above the sea level. The experiment was conducted in pots soil kept in vinyl house. Plants were grown in perforated plastic pots soil (drilled and lined with canvas). The pots were placed inside a bucket serving as water baths.

Based on screening at germination stage, relatively salt-tolerant eleven genotypes with two standard check variety BRRI dhan47 as tolerant and IR29 as susceptible (Islam et al., 2008) were used in this experiment. The genotypes were collected from Genetic Resources Unit of BSMRAU and Bangladesh Rice Research Institute (BRRI). The experiment was laid out in a complete randomized design (CRD) with four treatments where each treatment replicated five times. Four pre germinated seeds were sown at the soil surface of each pot (Gregorio et al., 1997) and finally one seedling was kept in each pot. Three levels of saline water with electrical conductivities at 5,10 and $15 \mathrm{dSm}^{-1}$ and ordinary tap water as the control made up a total of four salinity treatments. The pots were irrigated with ordinary tap water until 60 days of sowing. Then saline water collected from the coastal areas and it was diluted at 5,10 and $15 \mathrm{dSm}^{-1}$.After 60 days of seed sowing the treatment of saline water as control $\left(0.3 \mathrm{dSm}^{-1}\right), 5,10$ and $15 \mathrm{dSm}^{-1}$ was added in bucket.

However, before exposing the plants with the respective salinity treatments, they were exposed to $2.5 \mathrm{dSm}^{-1}$ salinity for 5 days to protect the 
plants from osmotic shock. Afterwards, plants of the treatments of 5,10 and $15 \mathrm{dSm}^{-1}$ were irrigated with $3.5,7.5$ and $12 \mathrm{dSm}^{-1}$ respectively for 7 days. Control plants were irrigated with tap water $\left(0.3 \mathrm{dSm}^{-1}\right)$. After application of saline water, the soil slowly absorbed the salt and the salinity was developed in the soil and finally attained an equilibrium condition between water and soil salinity. Soil salinity decreased gradually at the end of crop life cycle. Water level was maintained daily $(1 \mathrm{~cm}$ above soil surface) by adding ordinary tap water. BRRI recommended cultural practices like nutrient management, weed management, pest management were done as and when necessary (BRRI, 2017).

The crops were harvested at the time of maturity and data on plant height $(\mathrm{cm})$, total tillers per hill, effective tillers per hill, length of panicle, number of filled grains per panicle, 1000 seed weight and grain yield were recorded. 1000-seed were counted by Multi Auto Counter (Model DC 1 No. 102, Everwell Corporation, Tokyo, Japan) and weighed with an electronic balance. Seed yield of each pot was recorded and adjusted to $12 \%$ moisture content and mean was expressed on per plant basis. To check the salinity level gained by the soil at the end of the experiment, sampling of soil and saturation paste extract was carried out and soil salinity was measured after harvesting using EC meter.

\subsection{Reduction (\%) of plant characters}

The reduction (\%) of plant character was calculated with the following equation

Reduction $(\%)=$

Plant character at control - Plant character at saline condition

Plant character at control condition

\subsection{Statistical analysis}

The collected data were analyzed to assess their statistical significance. Statistix 10 program were used to perform statistical analysis. Means were separated by least significant difference (LSD).

\section{Results and Discussion}

\subsection{Plant height reduction}

Plant height is the most important character among the morpho-physiology which also acts as a key of shoot yield as well as total biomass production. Plant height reduction percent of different rice genotypes was influenced by different salinity level (Fig.1). At 10 and 15 $\mathrm{dSm}^{-1}$ salinity levels, the lowest plant height reduction $(3.84 \%$ and $7.77 \%$ ) was found in Soloi and the highest $(20.73 \%$ and $29.05 \%)$ was found in Awned-1. There was a wide variation among the genotypes. It has been reported that plant height decreased progressively with increase in salinity levels (Mahmmad et al., 2009). The influence of salinity on the height has been reported by many researchers (Falah, 2010; Rad et al., 2011).

\subsection{Number of total tiller per hill and total tiller reduction}

Different salinity levels had significant effect on number of total tiller per hill. Number of total tiller per hill of all genotypes decreased due to increasing salinity level. The maximum number of total tiller per hill of all genotypes was found in control condition and the minimum was at 15 $\mathrm{dSm}^{-1}$ salinity levels (Table 1 ). The maximum number of total tiller per hill $(28.20,27.20,25.20$ and 22.80 at control, $5 \mathrm{dSm}^{-1}, 10 \mathrm{dSm}^{-1}$ and 15 $\mathrm{dSm}^{-1}$ respectively) was found in Soloi. Due to salinity stress total tiller reduction was varied from $1.28 \%$ to $81.90 \%$ (Fig. 2). Other than check variety, the minimum total tiller reduction $(3.55 \%, 10.64 \%$ and $1 \times 10$ B\% at 5,10 and 15 $\mathrm{dSm}^{-1}$ respectively) was found in Soloi and the maximum was found in Gunshi (Fig. 2). Patnai 23 and Chapali also showed better result compared to other genotypes. Significant decrease in number of total tiller per hill has been reported by application of different level of salinity (Mahmmad et al., 2009; Rad et al., 2011). 


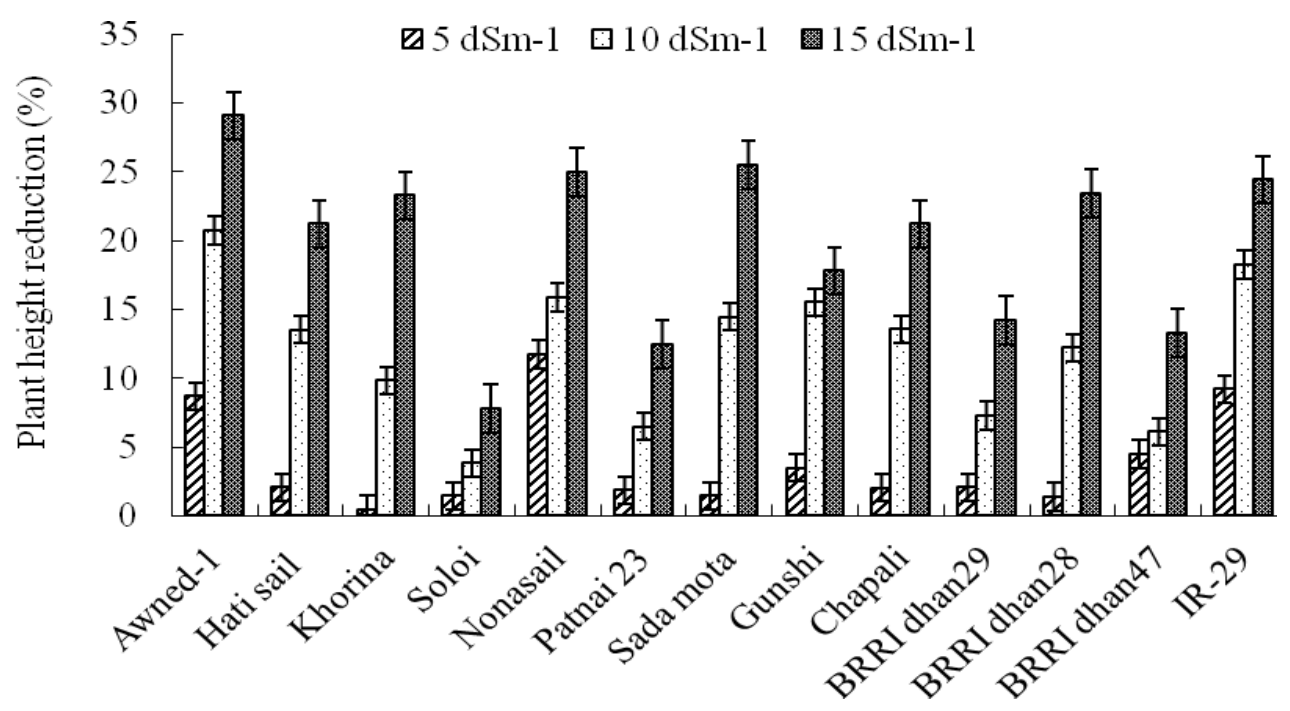

Figure 1.Plant height reduction (\%) of rice genotypes under varied salinity levels. Averages from three independent experiments are shown. Error bars represent the SE.

Table 1.Total tillerhill ${ }^{-1}$ of rice genotypes under different salinity levels

\begin{tabular}{lcccc}
\hline \multirow{2}{*}{ Genotype } & \multicolumn{4}{c}{ Total tiller/hill } \\
\cline { 2 - 5 } & $\begin{array}{c}\text { Control } \\
\left(0.3 \mathrm{dSm}^{-1}\right)\end{array}$ & $5 \mathrm{dSm}^{-1}$ & $10 \mathrm{dSm}^{-1}$ & $15 \mathrm{dSm}^{-1}$ \\
\hline Awned-1 & $20.80 \mathrm{de}^{\mathrm{A}}$ & $19.60 \mathrm{f}^{\mathrm{B}}$ & $17.40 \mathrm{de}^{\mathrm{C}}$ & $15.80 \mathrm{~d}^{\mathrm{D}}$ \\
Hati sail & $27.80 \mathrm{a}^{\mathrm{A}}$ & $24.20 \mathrm{~b}^{\mathrm{B}}$ & $20.00 \mathrm{~b}^{\mathrm{C}}$ & $17.60 \mathrm{~b}^{\mathrm{D}}$ \\
Khorina & $21.60 \mathrm{~d}^{\mathrm{A}}$ & $20.40 \mathrm{ef}^{\mathrm{B}}$ & $19.20 \mathrm{c}^{\mathrm{C}}$ & $16.80 \mathrm{c}^{\mathrm{D}}$ \\
Soloi & $28.20 \mathrm{a}^{\mathrm{A}}$ & $27.20 \mathrm{a}^{\mathrm{A}}$ & $25.20 \mathrm{a}^{\mathrm{B}}$ & $22.80 \mathrm{a}^{\mathrm{C}}$ \\
Nonasail & $23.80 \mathrm{c}^{\mathrm{A}}$ & $21.40 \mathrm{~cd}^{\mathrm{B}}$ & $16.60 \mathrm{f}^{\mathrm{C}}$ & $14.27 \mathrm{ef}^{\mathrm{D}}$ \\
Patnai 23 & $23.40 \mathrm{c}^{\mathrm{A}}$ & $21.80 \mathrm{c}^{\mathrm{B}}$ & $19.40 \mathrm{bc}^{\mathrm{C}}$ & $17.80 \mathrm{~b}^{\mathrm{D}}$ \\
Sadamota & $24.80 \mathrm{~b}^{\mathrm{A}}$ & $20.60 \mathrm{de}^{\mathrm{B}}$ & $17.60 \mathrm{~d}^{\mathrm{C}}$ & $14.80 \mathrm{e}^{\mathrm{D}}$ \\
Gunshi & $25.20 \mathrm{~b}^{\mathrm{A}}$ & $21.60 \mathrm{c}^{\mathrm{B}}$ & $15.60 \mathrm{~g}^{\mathrm{C}}$ & $8.60 \mathrm{i}^{\mathrm{D}}$ \\
Chapali & $20.40 \mathrm{e}^{\mathrm{A}}$ & $19.60 \mathrm{f}^{\mathrm{B}}$ & $16.90 \mathrm{ef}^{\mathrm{C}}$ & $15.88 \mathrm{~d}^{\mathrm{D}}$ \\
BRRI dhan29 & $20.60 \mathrm{e}^{\mathrm{A}}$ & $17.60 \mathrm{~g}^{\mathrm{B}}$ & $15.80 \mathrm{~g}^{\mathrm{C}}$ & $13.80 \mathrm{f}^{\mathrm{D}}$ \\
BRRI dhan28 & $16.60 \mathrm{f}^{\mathrm{A}}$ & $14.60 \mathrm{~h}^{\mathrm{B}}$ & $13.40 \mathrm{~h}^{\mathrm{C}}$ & $11.40 \mathrm{~h}^{\mathrm{D}}$ \\
BRRI dhan47 (check) & $15.60 \mathrm{~g}^{\mathrm{A}}$ & $15.40 \mathrm{~h}^{\mathrm{A}}$ & $13.20 \mathrm{~h}^{\mathrm{B}}$ & $12.88 \mathrm{~g}^{\mathrm{B}}$ \\
IR-29 (check) & $23.20 \mathrm{c}^{\mathrm{A}}$ & $14.80 \mathrm{~h}^{\mathrm{B}}$ & $10.00 \mathrm{i}^{\mathrm{C}}$ & $4.20 \mathrm{j}^{\mathrm{D}}$ \\
\hline CV (\%) & 4.19 & 3.69 & 3.42 & 3.82 \\
LSD (0.05) & 0.82 & 0.83 & 0.63 & 0.58 \\
\hline
\end{tabular}

Means with same small letter(s) in same column and superscript capitalletter(s) in same row are not differed significantly at $5 \%$ level of significance. 


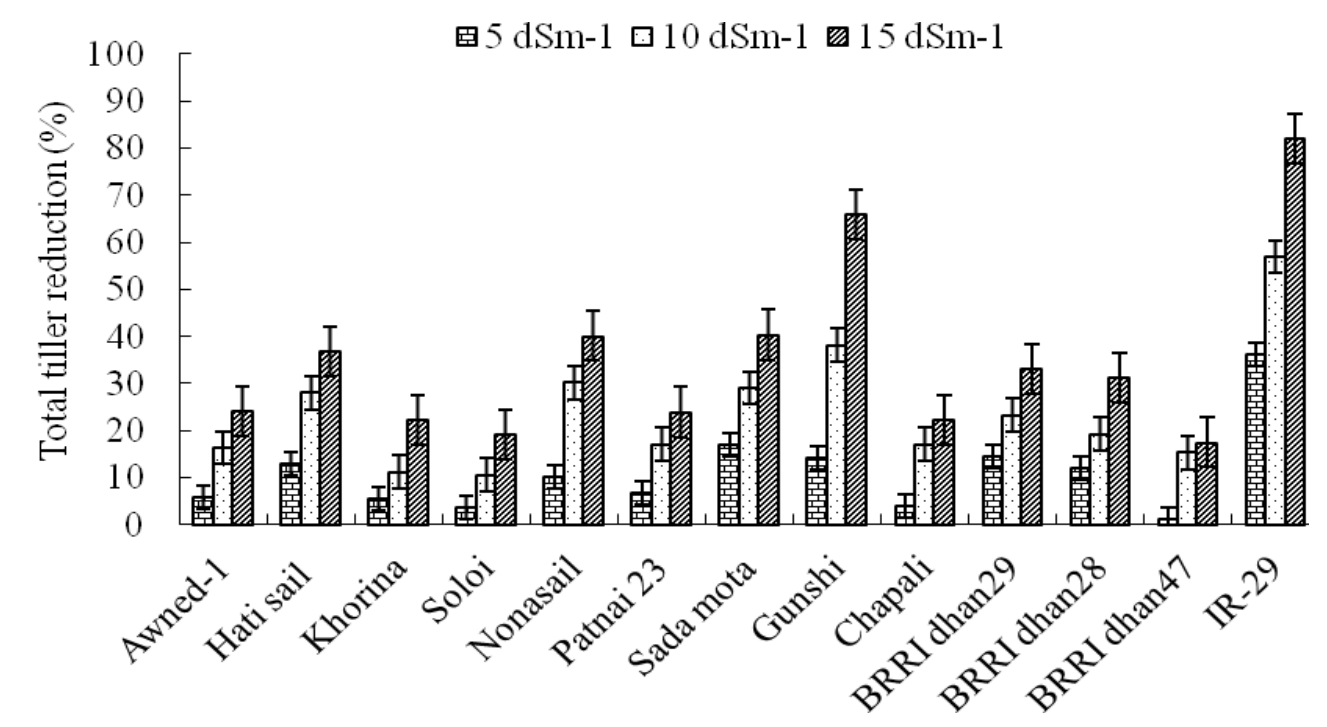

Figure 2. Total tiller reduction (\%) of rice genotypes under varied salinity levels. Averages from three independent experiments are shown. Error bars represent the SE.

Table 2. Effective tillerhill ${ }^{-1}$ of rice genotypes under different salinity levels

\begin{tabular}{|c|c|c|c|c|}
\hline \multirow[b]{2}{*}{ Genotype } & \multicolumn{4}{|c|}{ Effective tillers/hill } \\
\hline & $\begin{array}{c}\text { Control } \\
\left(0.3 \mathrm{dSm}^{-1}\right)\end{array}$ & $5 \mathrm{dSm}^{-1}$ & $10 \mathrm{dSm}^{-1}$ & $15 \mathrm{dSm}^{-1}$ \\
\hline Awned-1 & $16.56 \mathrm{~g}^{\mathrm{A}}$ & $14.60 \mathrm{f}^{\mathrm{B}}$ & $10.40 \mathrm{de}^{\mathrm{C}}$ & $5.60 \mathrm{f}^{\mathrm{D}}$ \\
\hline Hati sail & $18.60 \mathrm{e}^{\mathrm{A}}$ & $16.40 \mathrm{e}^{\mathrm{B}}$ & $10.52 \mathrm{de}^{\mathrm{C}}$ & $6.20 \mathrm{e}^{\mathrm{D}}$ \\
\hline Khorina & $15.00 \mathrm{~h}^{\mathrm{A}}$ & $9.00 \mathrm{i}^{\mathrm{B}}$ & $7.60 \mathrm{~g}^{\mathrm{C}}$ & $4.00 \mathrm{gh}^{\mathrm{D}}$ \\
\hline Soloi & $25.60 \mathrm{a}^{\mathrm{A}}$ & $24.20 \mathrm{a}^{\mathrm{B}}$ & $22.20 \mathrm{a}^{\mathrm{C}}$ & $16.20 \mathrm{a}^{\mathrm{D}}$ \\
\hline Nonasail & $18.60 \mathrm{e}^{\mathrm{A}}$ & $18.00 \mathrm{c}^{\mathrm{A}}$ & $9.40 \mathrm{f}^{\mathrm{B}}$ & $4.40 \mathrm{~g}^{\mathrm{C}}$ \\
\hline Patnai 23 & $17.40 \mathrm{f}^{\mathrm{A}}$ & $16.00 \mathrm{e}^{\mathrm{B}}$ & $13.80 \mathrm{c}^{\mathrm{C}}$ & $10.80 \mathrm{c}^{\mathrm{D}}$ \\
\hline Sadamota & $19.40 \mathrm{~d}^{\mathrm{A}}$ & $18.80 \mathrm{~b}^{\mathrm{B}}$ & $10.20 \mathrm{e}^{\mathrm{C}}$ & $6.20 \mathrm{e}^{\mathrm{D}}$ \\
\hline Gunshi & $20.60 c^{A}$ & $15.00 \mathrm{f}^{\mathrm{B}}$ & $9.60 \mathrm{f}^{\mathrm{C}}$ & $3.60 \mathrm{~h}^{\mathrm{D}}$ \\
\hline Chapali & $19.00 \mathrm{de}^{\mathrm{A}}$ & $17.40 \mathrm{~d}^{\mathrm{B}}$ & $15.40 \mathrm{~b}^{\mathrm{C}}$ & $12.00 \mathrm{~b}^{\mathrm{D}}$ \\
\hline BRRI dhan29 & $17.80 \mathrm{f}^{\mathrm{A}}$ & $10.80 \mathrm{~h}^{\mathrm{B}}$ & $7.20 \mathrm{~g}^{\mathrm{C}}$ & $4.40 \mathrm{~g}^{\mathrm{D}}$ \\
\hline BRRI dhan 28 & $16.20 \mathrm{~g}^{\mathrm{A}}$ & $9.20 \mathrm{i}^{\mathrm{B}}$ & $5.80 \mathrm{~h}^{\mathrm{C}}$ & $2.20 \mathrm{i}^{\mathrm{D}}$ \\
\hline BRRI dhan47 (check) & $14.50 \mathrm{~h}^{\mathrm{A}}$ & $13.40 \mathrm{~g}^{\mathrm{B}}$ & $10.80 \mathrm{~d}^{\mathrm{C}}$ & $9.60 \mathrm{~d}^{\mathrm{D}}$ \\
\hline IR-29 (check) & $21.60 \mathrm{~b}^{\mathrm{A}}$ & $10.80 \mathrm{~g}^{\mathrm{B}}$ & $4.20 \mathrm{i}^{\mathrm{C}}$ & $1.20 \mathrm{j}^{\mathrm{D}}$ \\
\hline $\mathrm{CV}(\%)$ & 4.47 & 5.21 & 4.56 & 4.46 \\
\hline $\operatorname{LSD}_{(0.05)}$ & 0.64 & 0.55 & 0.50 & 0.50 \\
\hline
\end{tabular}

Means with same small letter(s) in same column and superscript capital letter(s) in same row are not differed significantly at $5 \%$ level of significance. 


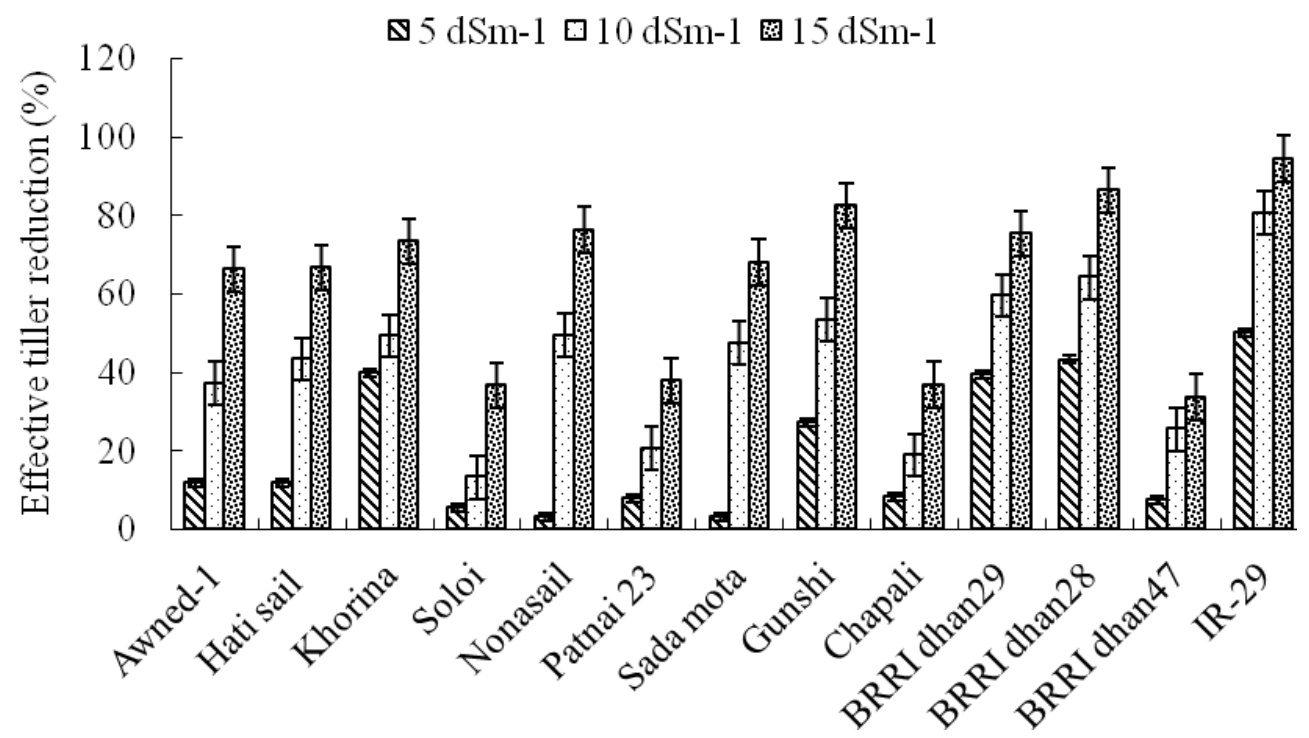

Figure 3. Effective tiller reduction (\%) of rice genotypes under varied salinity levels. Averages from three independent experiments are shown. Error bars represent the SE.

Table 3. Panicle length of rice genotypes under different salinity levels

\begin{tabular}{lcccc}
\hline & \multicolumn{4}{c}{ Panicle length $(\mathrm{cm})$} \\
\cline { 2 - 5 } Genotype & $\begin{array}{c}\text { Control } \\
\left(0.3 \mathrm{dSm}^{-1}\right)\end{array}$ & $5 \mathrm{dSm}^{-1}$ & $10 \mathrm{dSm}^{-1}$ & $15 \mathrm{dSm}^{-1}$ \\
\hline Awned-1 & $29.80 \mathrm{a}^{\mathrm{A}}$ & $25.37 \mathrm{ab}^{\mathrm{B}}$ & $22.30 \mathrm{~cd}^{\mathrm{C}}$ & $19.88 \mathrm{c}^{\mathrm{D}}$ \\
Hati sail & $24.26 \mathrm{de}^{\mathrm{A}}$ & $22.39 \mathrm{~d}^{\mathrm{B}}$ & $20.11 \mathrm{ef}^{\mathrm{C}}$ & $17.39 \mathrm{~d}^{\mathrm{D}}$ \\
Khorina & $28.82 \mathrm{ab}^{\mathrm{A}}$ & $26.50 \mathrm{a}^{\mathrm{B}}$ & $24.57 \mathrm{ab}^{\mathrm{C}}$ & $23.85 \mathrm{a}^{\mathrm{D}}$ \\
Soloi & $17.42 \mathrm{~h}^{\mathrm{A}}$ & $16.72 \mathrm{~g}^{\mathrm{AB}}$ & $15.85 \mathrm{~h}^{\mathrm{BC}}$ & $14.78 \mathrm{fg}^{\mathrm{C}}$ \\
Nonasail & $22.90 \mathrm{efg}^{\mathrm{A}}$ & $21.28 \mathrm{de}^{\mathrm{B}}$ & $19.31 \mathrm{fg}^{\mathrm{C}}$ & $17.22 \mathrm{~d}^{\mathrm{D}}$ \\
Patnai 23 & $23.86 \mathrm{def}^{\mathrm{A}}$ & $22.58 \mathrm{~cd}^{\mathrm{AB}}$ & $21.23 \mathrm{de}^{\mathrm{BC}}$ & $20.10 \mathrm{c}^{\mathrm{C}}$ \\
Sadamota & $27.18 \mathrm{bc}^{\mathrm{A}}$ & $26.12 \mathrm{a}^{\mathrm{A}}$ & $25.34 \mathrm{a}^{\mathrm{A}}$ & $22.67 \mathrm{ab}^{\mathrm{B}}$ \\
Gunshi & $27.82 \mathrm{~b}^{\mathrm{A}}$ & $25.85 \mathrm{a}^{\mathrm{AB}}$ & $24.63 \mathrm{ab}^{\mathrm{B}}$ & $21.32 \mathrm{bc}^{\mathrm{C}}$ \\
Chapali & $17.48 \mathrm{~h}^{\mathrm{A}}$ & $16.54 \mathrm{~g}^{\mathrm{AB}}$ & $15.75 \mathrm{~h}^{\mathrm{BC}}$ & $14.86 \mathrm{fg}^{\mathrm{C}}$ \\
BRRI dhan29 & $22.16 \mathrm{fg}^{\mathrm{A}}$ & $21.20 \mathrm{de}^{\mathrm{AB}}$ & $20.09 \mathrm{ef}^{\mathrm{B}}$ & $16.41 \mathrm{de}^{\mathrm{C}}$ \\
BRRI dhan28 & $22.74 \mathrm{efg}^{\mathrm{A}}$ & $20.02 \mathrm{e}^{\mathrm{B}}$ & $18.21 \mathrm{~g}^{\mathrm{C}}$ & $15.66 \mathrm{ef}^{\mathrm{D}}$ \\
BRRI dhan47 (check) & $25.42 \mathrm{~cd}^{\mathrm{A}}$ & $24.04 \mathrm{bc}^{\mathrm{AB}}$ & $23.47 \mathrm{bc}^{\mathrm{AB}}$ & $22.20 \mathrm{~b}^{\mathrm{B}}$ \\
IR-29 (check) & $21.62 \mathrm{~g}^{\mathrm{A}}$ & $18.52 \mathrm{f}^{\mathrm{B}}$ & $15.42 \mathrm{~h}^{\mathrm{C}}$ & $14.01 \mathrm{~g}^{\mathrm{D}}$ \\
\hline CV (\%) & 4.73 & 3.96 & 3.59 & 4.95 \\
LSD $(0.05)$ & 1.9 & 1.5 & 1.2 & 1.5 \\
\hline
\end{tabular}

Means with same small letter(s) in same column and superscript capital letter(s) in same row are not differed significantly at $5 \%$ level of significance. 


\subsection{Number of effective tiller per hill and effective tiller reduction}

Different salinity levels had significant effect on number of effective tiller per hill. The maximum number of effective tiller per hill of all genotypes was found in control condition and the minimum was at $15 \mathrm{dSm}^{-1}$ salinity levels (Table 2). At control, 5, 10 and $15 \mathrm{dSm}^{-1}$ salinity level, the maximum number of effective tiller per hill (25.60, 24.20, 22.20 and 16.20 respectively) was found in Soloi (Table 2). Due to salinity the effective tiller reduction was varied from 3.23 to $94.44 \%$ (Fig. 3). At all salinity levels, other than check variety, the lowest effective tiller reduction percent were found in Soloi, Chapali and Patnai 23 and the highest was found in BRRI dhan28 (Fig. 3).

3.4 Panicle length and panicle length reduction Different salinity levels had significant effect on panicle length of different rice genotypes. The highest panicle length of all genotypes was found in control condition and the lowest was at $15 \mathrm{dSm}^{-1}$ salinity levels (Table 3 ). These results show that increase in salinity decreases length of rice panicle. The least panicle length was 14.01 $\mathrm{cm}$ which obtained at $15 \mathrm{dSm}^{-1}$ salinity which had $35.20 \%$ decreases in compare with control treatment. Other than check variety, at $15 \mathrm{dSm}^{-1}$ salinity, the lowest panicle length reduction (14.99\%) was found in Chapali and the highest (33.29) at Awned-1(Fig. 4). High effectiveness of salinity on length of rice panicle has been reported by many researchers (Ali et al. 2009; Rad et al., 2012b). Soil salinity is a major environmental stress that adversely affects plant growth and metabolism. Salinity affects plant physiology through changes of the water and ionic status in the cells and reduced panicle length ( $\operatorname{Rad}$ et al., 2012a).

\subsection{Number of filled grain per panicle and filled grain reduction}

The number of filled grain per panicle is the most influential yield component, and most closely correlated with seed yield. Data regarding filled grain per panicle of rice as influenced by different salinity level is shown in table 4. Results showed that the highest number of filled grains per panicle (116.9) was found in BRRI dhan 28 at control and the lowest (52.45) was found in IR 29 at $15 \mathrm{dSm}^{-1}$ salinity which has $37.71 \%$ decrease in compare with control treatment. At $15 \mathrm{dSm}^{-1}$ salinity, the minimum filled grain reduction was found in Chapali and Soloi $(24.90 \%$ and $25.17 \%$ respectively) and the maximum (39.99\%) was found in BRRI dhan28 compared to that of check variety (Fig. 5). These results indicate that high salinity decreased filled grains per panicle of rice. Therefore, increased salinity resulted in increased total number of empty grains per panicles and finally it decreases yield. Increased number of empty grains might be a result of assimilate shortage during grain filling, brought about by early leaf senescence caused in this case by salinity (Aref and Rad, 2012). Sterility and reduction in seed set were primarily due to reduced translocation of soluble carbohydrates to primary and secondary spikelets, accumulation of more sodium and less potassium in all floral parts and inhibition of the specific activity of starch synthetase in developing rice grains, thus reducing seed set (Aref and Rad, 2012). It was also reported that reducing seed set in the panicle, possibly as a consequence of decreased pollen viability which is greatly influenced by the ionic toxicity under salinity (Mohammadi Nejada et al., 2010).

\subsection{Thousand seeds weight reduction}

Seed weight is an important quality attribute of crop. Thousand seed weight reduction percent of different rice genotypes was influenced by different salinity level (Fig. 6). However, the highest 1000 seeds weight reduction (10.63\%) was found in BRRI dhan29 and the lowest (6.99\%) was found in Chapali at $15 \mathrm{dSm}^{-1}$ salinity other than the check variety (Fig. 6). High influence of salinity on the weight of 1000 seeds was reported by $\operatorname{Rad}$ et al., (2011). Presumably salinity reduces the contents of photosynthetic pigments and soluble proteins in the ovaries. This change might cause the decline of ovary photosynthesis leading to poor sugar production in the ovaries. 


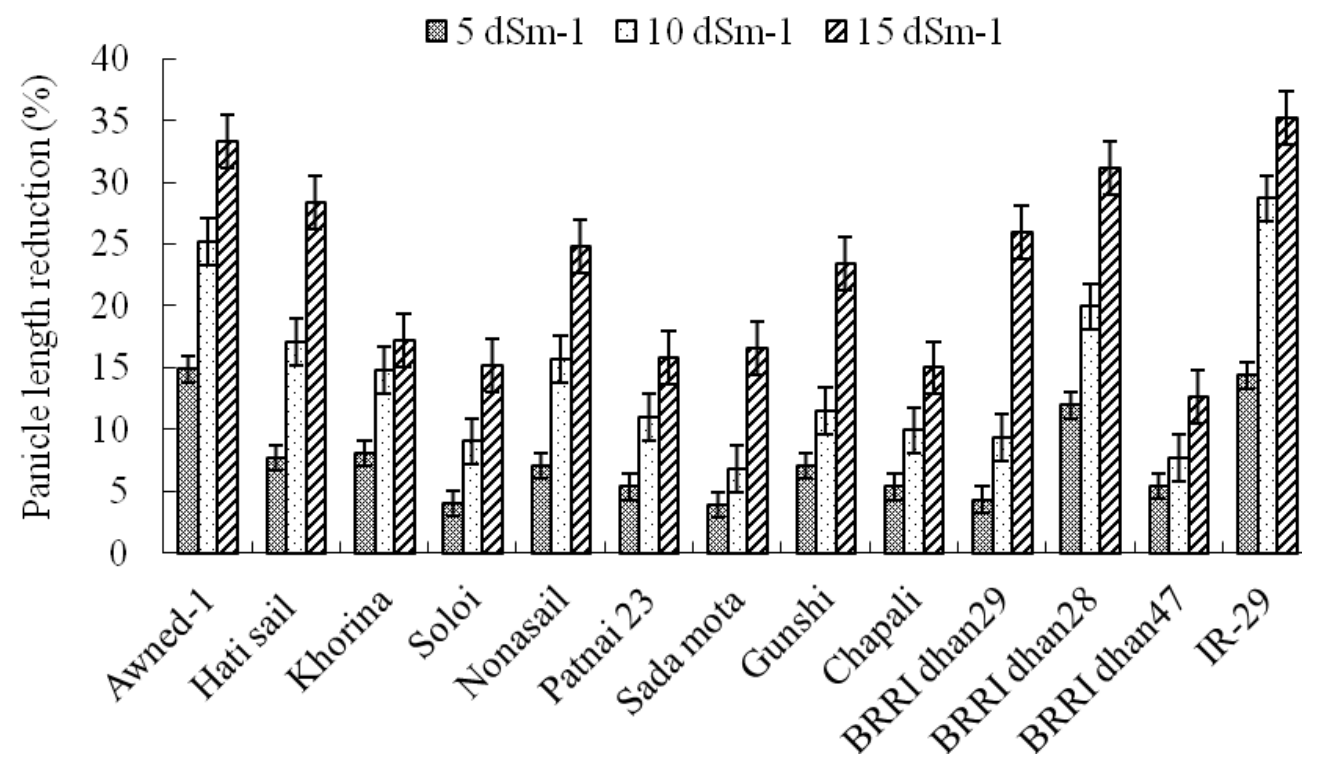

Figure 4. Panicle length reduction (\%) of rice genotypes under varied salinity levels. Averages from three independent experiments are shown. Error bars represent the SE.

Table 4. No. of filled grainspanicle ${ }^{-1}$ of rice genotypes under different salinity levels

\begin{tabular}{lcccc}
\hline & \multicolumn{4}{c}{ No. of filled grains/panicle } \\
\cline { 2 - 5 } Genotype & $\begin{array}{c}\text { Control } \\
\left(0.3 \mathrm{dSm}^{-1}\right)\end{array}$ & $5 \mathrm{dSm}^{-1}$ & $10 \mathrm{dSm}^{-1}$ & $15 \mathrm{dSm}^{-1}$ \\
\hline Awned-1 & $110.01 \mathrm{~b}^{\mathrm{A}}$ & $95.71 \mathrm{~b}^{\mathrm{B}}$ & $83.27 \mathrm{~b}^{\mathrm{C}}$ & $72.44 \mathrm{~b}^{\mathrm{D}}$ \\
Hati sail & $80.82 \mathrm{de}^{\mathrm{A}}$ & $70.31 \mathrm{ef}^{\mathrm{B}}$ & $61.17 \mathrm{~d}^{\mathrm{C}}$ & $55.22 \mathrm{ef}^{\mathrm{C}}$ \\
Khorina & $85.13 \mathrm{~d}^{\mathrm{A}}$ & $74.06 \mathrm{c}^{\mathrm{B}}$ & $64.43 \mathrm{~cd}^{\mathrm{C}}$ & $56.06 \mathrm{def}^{\mathrm{D}}$ \\
Soloi & $82.37 \mathrm{de}^{\mathrm{A}}$ & $75.67 \mathrm{cde}^{\mathrm{B}}$ & $65.35 \mathrm{~cd}^{\mathrm{C}}$ & $61.64 \mathrm{~cd}^{\mathrm{C}}$ \\
Nonasail & $91.20 \mathrm{c}^{\mathrm{A}}$ & $80.24 \mathrm{c}^{\mathrm{B}}$ & $67.50 \mathrm{c}^{\mathrm{C}}$ & $61.41 \mathrm{~cd}^{\mathrm{C}}$ \\
Patnai 23 & $78.80 \mathrm{e}^{\mathrm{A}}$ & $71.60 \mathrm{def}^{\mathrm{B}}$ & $60.80 \mathrm{~d}^{\mathrm{C}}$ & $58.32 \mathrm{de}^{\mathrm{C}}$ \\
Sadamota & $81.88 \mathrm{de}^{\mathrm{A}}$ & $71.21 \mathrm{def}^{\mathrm{B}}$ & $60.98 \mathrm{~d}^{\mathrm{C}}$ & $53.52 \mathrm{ef}^{\mathrm{D}}$ \\
Gunshi & $80.73 \mathrm{de}^{\mathrm{A}}$ & $68.70 \mathrm{f}^{\mathrm{B}}$ & $60.91 \mathrm{~d}^{\mathrm{C}}$ & $54.16 \mathrm{ef}^{\mathrm{D}}$ \\
Chapali & $85.63 \mathrm{~cd}^{\mathrm{A}}$ & $77.59 \mathrm{~cd}^{\mathrm{B}}$ & $69.03 \mathrm{c}^{\mathrm{C}}$ & $64.31 \mathrm{c}^{\mathrm{C}}$ \\
BRRI dhan29 & $114.43 \mathrm{ab}^{\mathrm{A}}$ & $101.51 \mathrm{ab}^{\mathrm{B}}$ & $85.67 \mathrm{~b}^{\mathrm{C}}$ & $70.62 \mathrm{~b}^{\mathrm{D}}$ \\
BRRI dhan28 & $116.90 \mathrm{a}^{\mathrm{A}}$ & $101.66 \mathrm{ab}^{\mathrm{B}}$ & $85.45 \mathrm{~b}^{\mathrm{C}}$ & $70.15 \mathrm{~b}^{\mathrm{D}}$ \\
BRRI dhan47 (check) & $112.53 \mathrm{ab}^{\mathrm{A}}$ & $105.90 \mathrm{a}^{\mathrm{A}}$ & $95.31 \mathrm{a}^{\mathrm{B}}$ & $85.10 \mathrm{a}^{\mathrm{C}}$ \\
IR-29 (check) & $84.21 \mathrm{de}^{\mathrm{A}}$ & $73.26 \mathrm{def}^{\mathrm{B}}$ & $60.74 \mathrm{~d}^{\mathrm{C}}$ & $52.45 \mathrm{f}^{\mathrm{D}}$ \\
\hline CV (\%) & 3.81 & 4.81 & 4.94 & 5.36 \\
LSD $(0.05)$ & 5.9 & 6.6 & 5.8 & 5.6 \\
\hline
\end{tabular}

Means with same small letter(s) in same column and superscript capital letter(s) in same row are not differed significantly at $5 \%$ level of significance. 


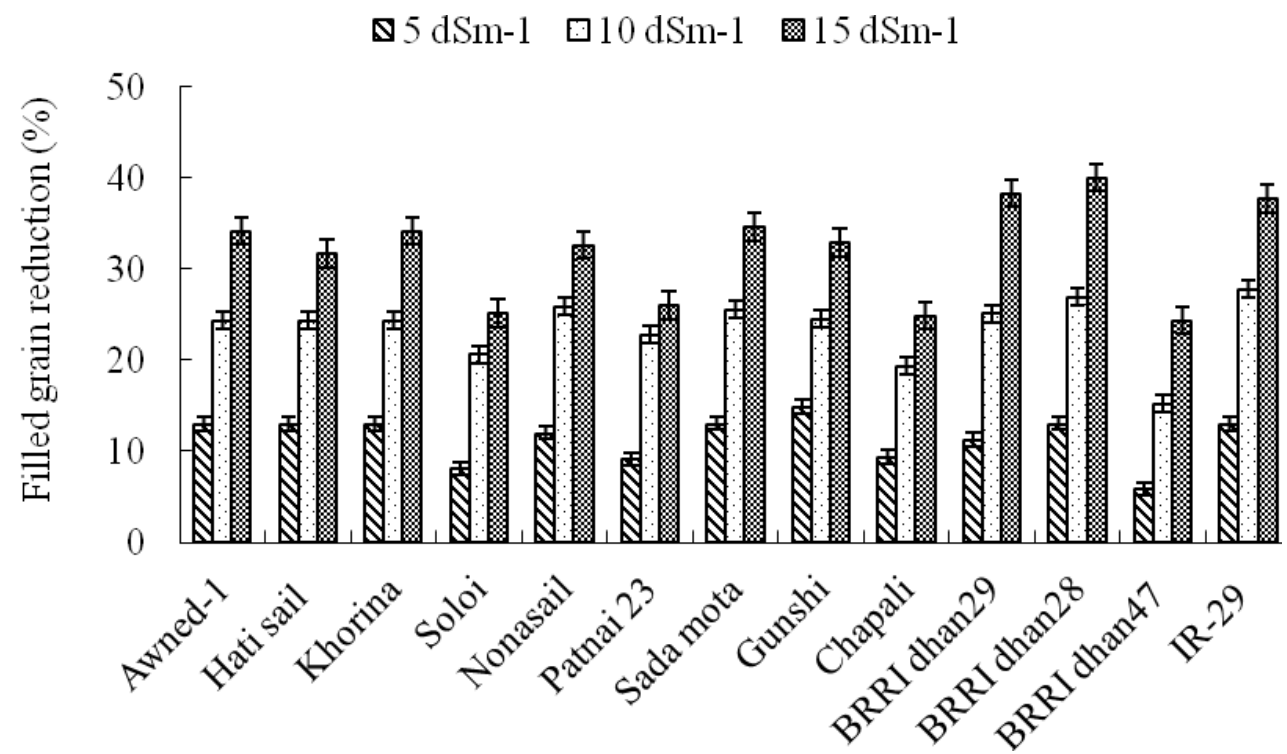

Figure 5. Filled grain reduction $(\%)$ of rice genotypes under varied salinity levels. Averages from three independent experiments are shown. Error bars represent the SE.

Table 5. Seed yield of rice genotypes under different salinity levels

\begin{tabular}{lcccc}
\hline & \multicolumn{4}{c}{ Seed yield $(\mathrm{g} / \mathrm{pot})$} \\
\cline { 2 - 5 } Genotype & $\begin{array}{c}\text { Control } \\
\left(0.3 \mathrm{dSm}^{-1}\right)\end{array}$ & $5 \mathrm{dSm}^{-1}$ & $10 \mathrm{dSm}^{-1}$ & $15 \mathrm{dSm}^{-1}$ \\
\hline Awned-1 & $31.91 \mathrm{e}^{\mathrm{A}}$ & $23.88 \mathrm{c}^{\mathrm{B}}$ & $14.44 \mathrm{~d}^{\mathrm{C}}$ & $6.60 \mathrm{~d}^{\mathrm{D}}$ \\
Hati sail & $19.51 \mathrm{~h}^{\mathrm{A}}$ & $14.60 \mathrm{f}^{\mathrm{B}}$ & $7.95 \mathrm{~h}^{\mathrm{C}}$ & $4.12 \mathrm{fg}^{\mathrm{D}}$ \\
Khorina & $27.95 \mathrm{f}^{\mathrm{A}}$ & $14.24 \mathrm{f}^{\mathrm{B}}$ & $10.21 \mathrm{~g}^{\mathrm{C}}$ & $4.56 \mathrm{ef}^{\mathrm{D}}$ \\
Soloi & $31.00 \mathrm{e}^{\mathrm{A}}$ & $26.15 \mathrm{~b}^{\mathrm{B}}$ & $20.43 \mathrm{c}^{\mathrm{C}}$ & $13.65 \mathrm{c}^{\mathrm{D}}$ \\
Nonasail & $34.13 \mathrm{~d}^{\mathrm{A}}$ & $27.85 \mathrm{~b}^{\mathrm{B}}$ & $12.07 \mathrm{f}^{\mathrm{C}}$ & $5.00 \mathrm{e}^{\mathrm{D}}$ \\
Patnai 23 & $36.89 \mathrm{c}^{\mathrm{A}}$ & $30.08 \mathrm{a}^{\mathrm{B}}$ & $21.52 \mathrm{~b}^{\mathrm{C}}$ & $15.76 \mathrm{~b}^{\mathrm{D}}$ \\
Sadamota & $22.15 \mathrm{~g}^{\mathrm{A}}$ & $18.22 \mathrm{e}^{\mathrm{B}}$ & $8.26 \mathrm{~h}^{\mathrm{C}}$ & $4.30 \mathrm{f}^{\mathrm{D}}$ \\
Gunshi & $34.68 \mathrm{~d}^{\mathrm{A}}$ & $20.98 \mathrm{~d}^{\mathrm{B}}$ & $11.62 \mathrm{f}^{\mathrm{C}}$ & $3.76 \mathrm{~g}^{\mathrm{D}}$ \\
Chapali & $37.73 \mathrm{c}^{\mathrm{A}}$ & $30.56 \mathrm{a}^{\mathrm{B}}$ & $23.49 \mathrm{a}^{\mathrm{C}}$ & $16.65 \mathrm{a}^{\mathrm{D}}$ \\
BRRI dhan29 & $47.91 \mathrm{a}^{\mathrm{A}}$ & $23.91 \mathrm{c}^{\mathrm{B}}$ & $13.23 \mathrm{e}^{\mathrm{C}}$ & $6.53 \mathrm{~d}^{\mathrm{D}}$ \\
BRRI dhan28 & $42.52 \mathrm{~b}^{\mathrm{A}}$ & $19.73 \mathrm{de}^{\mathrm{B}}$ & $10.33 \mathrm{~g}^{\mathrm{C}}$ & $3.20 \mathrm{~h}^{\mathrm{D}}$ \\
BRRI dhan47 (check) & $36.20 \mathrm{~cd}^{\mathrm{A}}$ & $30.73 \mathrm{a}^{\mathrm{B}}$ & $21.76 \mathrm{~b}^{\mathrm{C}}$ & $16.86 \mathrm{a}^{\mathrm{D}}$ \\
IR-29 (check) & $37.71 \mathrm{c}^{\mathrm{A}}$ & $15.45 \mathrm{f}^{\mathrm{B}}$ & $4.81 \mathrm{i}^{\mathrm{C}}$ & $1.16 \mathrm{i}^{\mathrm{D}}$ \\
\hline CV (\%) & 3.67 & 4.68 & 3.44 & 3.85 \\
LSD $(0.05)$ & 2.1 & 1.8 & 0.80 & 0.51 \\
\hline
\end{tabular}

Means with same small letter(s) in same column and superscript capital letter(s) in same row are not differed significantly at $5 \%$ level of significance. 


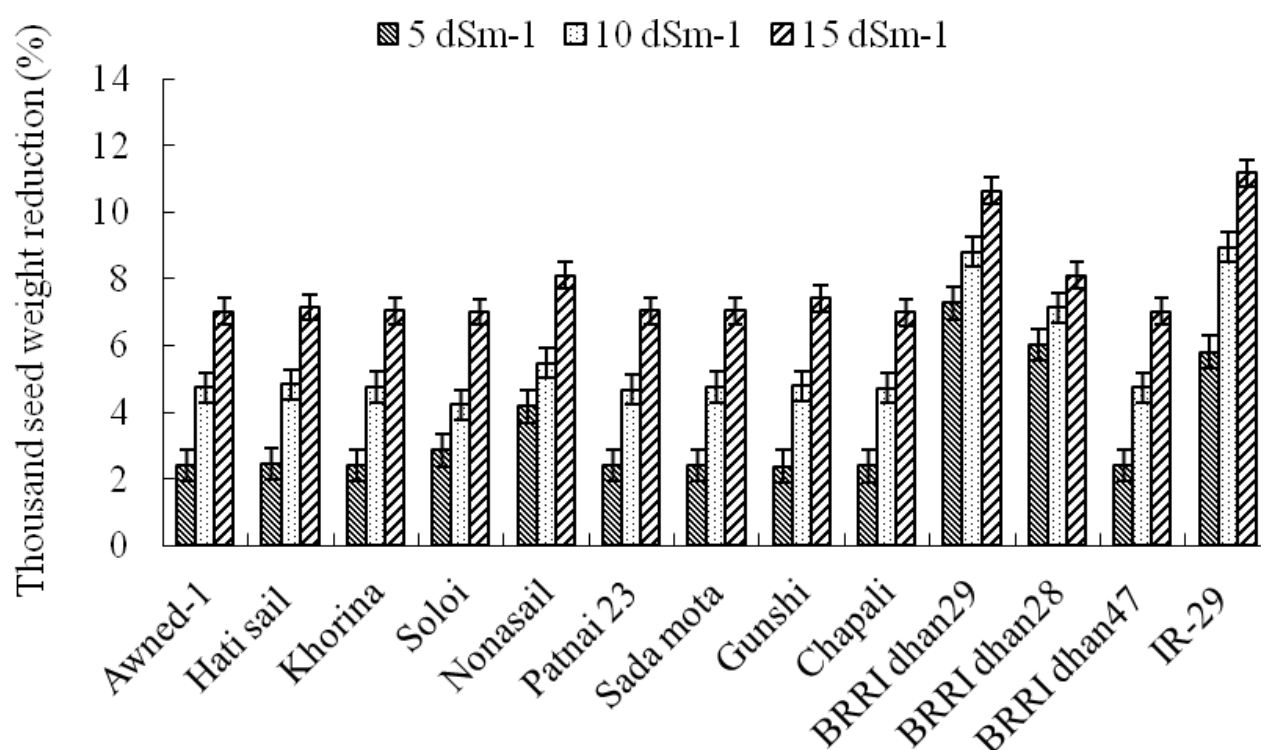

Figure 6. Thousand seed weight reduction $(\%)$ of rice genotypes under varied salinity levels. Averages from three independent experiments are shown. Error bars represent the SE.

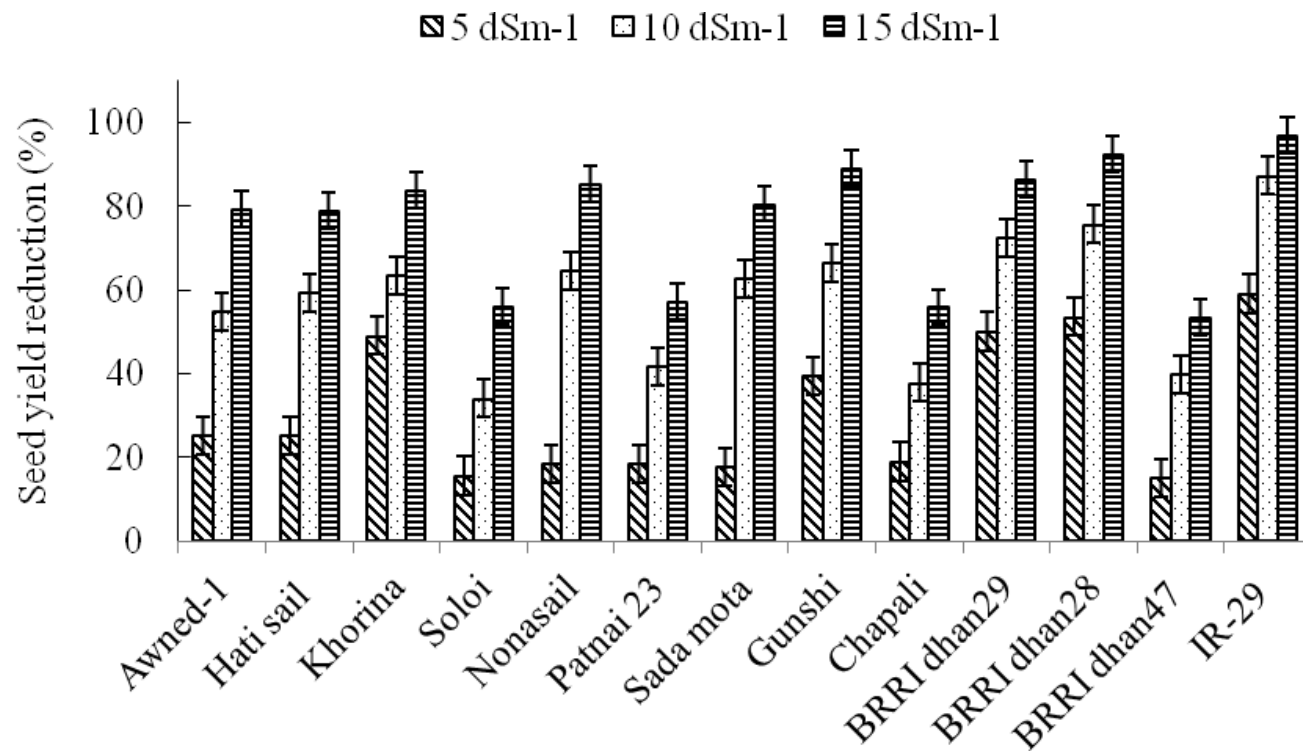

Figure 7. Seed yield reduction (\%) of rice genotypes under varied salinity levels. Averages from three independent experiments are shown. Error bars represent the SE. 
A reduced number of fertile florets and a lower rate of assimilate translocation from shoot to panicles are responsible for the lower dry weight of panicles and grain. This might be attributable to the rapid reduction in leaf photosynthesis, which is related to the decrease in photosynthetic pigments. Therefore, translocation of assimilates from stem to grain is the main source as well as limiting factors for growth and development of seeds.

\subsection{Seed yield and seed yield reduction}

The results from Table 5 indicated that seed yield of rice was significantly influenced by different salinity level. The maximum seed yields of all genotypes were found in control and the minimum were at $15 \mathrm{dSm}^{-1}$ salinity (Table 5). At control, the maximum seed yield (47.91 g/pot) was found in BRRI dhan29 while at 15 $\mathrm{dSm}^{-1}$ salinity, the maximum seed yield (16.65 $\mathrm{g} / \mathrm{pot})$ was found in Chapali and the minimum (3.18 g/pot) was found in BRRI dhan28 other than check variety. Patnai 23 (15.76 g/pot) and Soloi $(13.65 \mathrm{~g} / \mathrm{pot})$ also showed better seed yield at $15 \mathrm{dSm}^{-1}$ salinity (Table 5). Other than the check variety, the minimum seed yield reduction (55.88\%) was found in Chapali and the maximum $(92.51 \%)$ was found in BRRI dhan28 at $15 \mathrm{dSm}^{-1}$ salinity level (Fig. 7). Soloi (55.96\%) and Patnai $23(57.29 \%)$ also showed better result compared to other genotypes. It has long been recognized that a crop's sensitivity to salinity varies from one developmental growth stage to the next (Lauchli and Grattan, 2007). Rice has been reported as being salt-sensitive in both its vegetative and reproductive stages (Moradi and Ismail, 2007), leading to a reduction in productivity of more than $50 \%$ when exposed to $6.65 \mathrm{dSm}^{-1}$ salinity (Cha-um and Kirdmanee, 2010). Probably, the affected tillers number per hill, 1000 seeds weight and number of grain per panicle attributed for the reduced grain yield.

\section{Conclusions}

It could be concluded that salinity stress decreased plant height, number of total and effective tillers per hill, panicle length, number of filled grains per panicle, 1000- seed weight and finally, grain yield of rice. The study revealed that the rice genotypes Chapali, Patnai 23 and Soloi could adapt to salinity of $15 \mathrm{dSm}^{-1}$.

\section{Acknowledgement}

We are most grateful to Research Management Committee (RMC) of BSMRAU, Gazipur, Bangladesh for financial support to complete this research.

\section{References}

Abdallah, M.M.S., Abdelgawad, Z.A. and ElBassiouny, H.M.S. 2016. Alleviation of the adverse effects of salinity stress using trehalose in two rice varieties. South African Journal of Botany, 103: 275-282.

Ali, Y., Aslam, Z., Ashraf, Y. and Tahir, G. R. 2009. Effect of salinity on chlorophyll concentration, leaf area, yield and yield components of rice genotypes grown under saline environment. International Journal of Environmental Science and Technology, 1(3):221-225.

Aref, F. and Rad, H.E. 2012. Physiological characterization of rice under salinity stress during vegetative and reproductive stages. Indian Journal of Science and Technology, 5 (4): 2578-2586.

BRRI, 2017. Modern Rice Cultivation, Bangladesh Rice Research Institute. $20^{\text {th }}$ edition.

Cha-um, S. and Kirdmanee, C. 2010.Effect of glycinebetaine on proline, water use and photosynthetic efficiencies and growth of rice seedlings under salt stress. Turkish Journal of Agriculture and Forestry, 34: 517-527.

Darwish, E., Testerink, C., Khalil, M., El-Shihy, O. and Munnik, T. 2009. Phospholipid signaling responses in salt-stressed rice leaves. Plant and Cell Physiology, 50: 986-997. 
FAO, 2008.Land and Plant Nutrition Management Service. Retrieved from: http://www.fao.org/agb/agl/agll/spush/.

Falah A. et al. 2010. The effects of salinity at different growth stage on rice. Proceeding of 11th national congress opn agronomy. 2010.

Gregorio, G.B., D. Senadhira, And R.D. Mendoza, 1997. Screening rice for salinity tolerance. International Rice Research Institute, P.O. Box 933, Manila 1099, Philippines. IRR1 Discussion Paper Series no. 22.pp 1-28.

Haque, S.A. 2006. Salinity Problems and Crop Production in Coastal Regions of Bangladesh. Pakistan Journal of Botany, 38(5): 1359-1365.

Islam, M.R., Salam, M.A., Bhuiyan, M.A.R., Rahman, M.A., Yasmeen, R., Rahman, M.S., Uddin, M.K., Gregorio, G.B. and Ismail, A.M. 2008. BRRIdhan47: A salt tolerant rice variety for Boro season isolated through participatory variety selection. International Journal of Biological Research, 5 (1): 1-6.

Kamyab-Talesh, F., Mousavi, S.F., Asadi, R., Rezaei, M. and Khaledian, M.R. 2014. Evaluation of some rice cultivars' response to salinity stress using resistance indices. Archives of Agronomy and Soil Science,

DOI:

10.1080/03650340.2014.891730.

Lauchli, A. and Grattan, S.R. 2007.Plant Growth and Development under Salinity Stress. In: Jenks, M.A., P.M. Hasegawa and S.M. Jain, (Eds.), Advances in Molecular Breeding Toward Drought and Salt Tolerant Crops. Springer, Dordrecht, The Netherlands, pp: 1-32.

Ma, H., Chong, K. and Deng, X.W. 2007. Rice research: Past, present and future. Journal of Integrative Plant Biology, 49: 729-730.

Mahmmad, A., Latif, T. and Khan, M.A. 2009. Effect of salinity on growth, yield and yield components in Basmati rice germplasm. Pakistan Journal of Botany, 41(6): 3035-3045.

MohammadiNejada, G., Singhb, R.K., Arzanic, A., Rezaiec, A.M., Sabourid, H. and Gregoriob, G.B. 2010.Evaluation of salinity tolerance in rice genotypes. International Journal of Plant Production, 4(3): 199-207.

Moradi, F. and Ismail, A.M. 2007.Responses of photosynthesis, chlorophyll fluorescence and ROS scavenging systems to salt stress during seedling and reproductive stages in rice. Annals of Botany, 99: 1161-1173.

Munns, R. and Tester, M. 2008. Mechanisms of salinity tolerance. Annual Review of Plant Biology,59:651-681.

Quintero, J.M., Fournier J.M. and Benlloch, M. 2007. $\mathrm{Na}^{+}$accumulation in shoot is related to water transport in $\mathrm{K}^{+}$-starved sunflower plants but not in plants with a normal $\mathrm{K}^{+}$ status. Journal of Plant Physiology, 164: 60-67.

Rad, H.E, Aref, F. and Rezaei, M. 2012b. Rice Growth and Yield Components Respond to Changes in Water Salinity Stress. World Applied Sciences Journal, 20 (7): 997-1007.

Rad, H.E, Aref, F., Rezaei, M., Amiri, E. and Khaledian, M.R. 2011. The effects of salinity at different growth stage on rice yield. Ecology Environment \& Conservation, 17 (2): 111-117.

Rad, H.E., Aref, F. and Rezaei, M. 2012a.Response of Rice to Different Salinity Levels during Different Growth Stages. Research Journal of Applied Sciences, Engineering and Technology, 4(17): 3040-3047.

Reddy, I.N.B.L., Kim, B.K., Yoon, I.S., Kim, K.H. and Kwon, T.R 2017.Salt Tolerance in Rice: Focus on Mechanisms and Approaches. Rice Science, 24(3): 123144. 
Silva, C., Martinez, V. and Carvajal, M. 2008. Osmotic versus toxic effects of $\mathrm{NaCl}$ on pepper plants. Biologia Plantarum, 52 (1): 72-79.

Siringam, K., Juntawong, N., Cha-um, S. and Kirdmanee, C. 2011. Salt stress induced ion accumulation, ion homeostasis, membrane injury and sugar contents in saltsensitive rice (Oryza sativa L. spp. indica) roots under is oosmotic conditions. African Journal of Biotechnology, 10: 1340-1346.

UNFPA, 2014.State of World Population 2014. http://www.unfpa.org/swp/200/ 